\title{
Le débat sur l'Union anglo-écossaise : la dernière phase (1706-1707)
}

\section{Yannick Deschamps}

\section{(2) OpenEdition}

\section{Journals}

\section{Édition électronique}

URL : http://journals.openedition.org/etudesecossaises/3493

DOI : 10.4000/etudesecossaises.3493

ISSN : 1969-6337

\section{Éditeur}

UGA Éditions/Université Grenoble Alpes

\section{Édition imprimée}

Date de publication : 1 mars 2001

Pagination : 195-207

ISBN : 978-2-84310-198-4

ISSN : 1240-1439

\section{Référence électronique}

Yannick Deschamps, "Le débat sur l'Union anglo-écossaise : la dernière phase (1706-1707) », Études écossaises [En ligne], 7 | 2001, mis en ligne le 29 mars 2018, consulté le 08 septembre 2020. URL : http://journals.openedition.org/etudesecossaises/3493 ; DOl : https://doi.org/10.4000/ etudesecossaises.3493 


\section{Le débat sur l'Union anglo-écossaise La dernière phase (1706-1707)}

YANNICK Deschamps Université de Paris 12.
À la veille de l'Union, l'Angleterre et l'Écosse se trouvent dans une situation constitutionnelle originale: elles constituent deux royaumes distincts et indépendants, mais elles sont placées sous l'autorité d'un monarque commun. Il en va ainsi - la période du Commonwealth mise à part - depuis l'Union des couronnes, réalisée en 1603 par Jacques VI-I ${ }^{\text {er }}$.

Cette Union est mise à l'épreuve dès le début du XVIII siècle. En 1700, l'unique enfant d'Anne Stuart, le duc de Gloucester, meurt. Pour éviter une crise dynastique, le Parlement anglais vote l'Act of Settlement (1701), en vertu duquel, à la mort d'Anne, la couronne anglaise reviendra à Sophie de Hanovre. On s'attend, à Londres, à ce que la succession hanovrienne soit entérinée par le Parlement écossais. Mais celui-ci entend réaffirmer son indépendance à l'égard de l'Angleterre, qui, par le truchement de la prérogative royale, ne cesse de s'ingérer dans les affaires de l'Écosse. En 1703, au cours d'une session mémorable dominée par Fletcher, «le Patriote», le Parlement écossais vote l'Act of Security, qui menace d'écarter la Maison de Hanovre du trône d'Écosse si les libertés fondamentales du peuple écossais ne sont pas garanties. L'Angleterre craint alors que sa voisine septentrionale n'échappe à son influence et ne se rapproche de la France. Sa riposte est à la mesure de son inquiétude: les Écossais sont sommés d'adopter la succession hanovrienne ou de s'engager à entamer des négociations d'union avant le 25 décembre 1705 . S'ils refusent de s'exécuter, ils seront déclarés étrangers et leurs exportations de toile, de bétail et de charbon seront interdites en Angleterre. L'Alien Act (5 février 1705) déclenche en Écosse une vague d'anglophobie sans précédent. Toutefois, le Parlement écossais finit par céder au chantage exercé par Londres. Le 25 septembre 1705, il accepte le principe d'une union avec l'Angleterre. 
Les négociations d'union, qui se tiennent à Whitehall, commencent le 16 avril 1706. Le 25 février, les commissaires écossais se prononcent en faveur d'une «union par incorporation" et d'un parlement britannique unique. Ils approuvent également la succession hanovrienne. En contrepartie, l'Écosse acquiert le droit de commercer librement avec l'Angleterre et ses colonies. Le 23 juillet, les commissaires anglais et écossais présentent les 25 articles du traité d'Union à la reine Anne.

Pour avoir force de loi, le traité doit recevoir l'assentiment des Parlements anglais et écossais. Il est d'abord soumis au Parlement d'Édimbourg, censé être moins favorable à sa ratification. Les débats commencent le 3 octobre. Les principales clauses du traité, tenues secrètes pendant la durée des négociations, ne tardent pas à filtrer. En tout état de cause, elles sont rendues publiques le 10 octobre. Le peuple écossais réagit avec indignation et colère lorsqu'il apprend que le traité prévoit une «union par incorporation», et donc la suppression du Parlement écossais. Au sein du Parlement, toutefois, les unionistes disposent d'une majorité confortable. L'esprit d'indépendance manifesté en 1703 n'a pas résisté aux pressions de la Cour: octroi de titres et de sinécures, règlement opportun d'arrérages, sanctions à l'encontre des parlementaires indociles. Le 16 janvier 1707, le traité d'Union est entériné par le Parlement écossais. Le 4 mars, il est ratifié à Westminster. L'Union entre en vigueur le $1^{\text {er }}$ mai.

Entre 1700 et 1707, l'Union est la question qui suscite le plus d'intérêt en Écosse. De nombreux pamphlets, sermons et poèmes lui sont consacrés. Cette étude traitera plus particulièrement des écrits publiés pendant les années 1706-1707, entre le début des négociations d'union et la ratification du traité par le Parlement écossais. Elle exposera les arguments avancés par les pamphlétaires anti-unionistes et par leurs rivaux unionistes. Puis elle s'efforcera d'évaluer le degré de sincérité de ces arguments et de mesurer - autant que faire se peut - leur influence sur les mentalités écossaises.

Les polémistes qui écrivent sur l'Union en 1706-1707 n'ont pas le recul nécessaire pour en peser les avantages et les inconvénients. Soit ils cherchent à la promouvoir, soit ils tentent de la discréditer. Les principaux auteurs anti-unionistes sont James Hodges et George Ridpath, deux Whigs écossais domiciliés à Londres. Ils sont secondés par les ministres presbytériens James Webster, William Wright et Robert Wyllie. La cause 
anti-unioniste peut également compter sur la verve de Lord Belhaven, l'une des grandes figures du parti de la Cour, et sur la plume acerbe de l'ancien médecin de Jacques II, Patrick Abercromby. Elle reçoit en outre le soutien de William Black, manufacturier et avocat de son état. Andrew Fletcher, le "Patriote», tempête contre l'Union à la Chambre, mais il ne prend pas la plume. Son dernier ouvrage sur le sujet remonte à $1704^{1}$.

Du côté unioniste, William Seton et John Clerk, tous deux juristes, parlementaires et commissaires chargés de négocier le traité, ne ménagent pas leurs efforts. Ils confèrent au débat sérieux et compétence. Lord Cromarty met au service de l'Union son enthousiasme et sa grande expérience politique; John Arbuthnot, sa finesse et son talent rhétorique. Mais l'homme qui apporte le soutien le plus précieux à la cause unioniste est sans conteste Daniel Defoe. Envoyé par Harley à Édimbourg pour promouvoir l'Union, il remplit avec énergie et talent la mission qui lui a été confiée.

Quels sont les arguments des uns et des autres? Les auteurs anti-unionistes sont unanimes à déplorer la perte de la souveraineté écossaise. L'Union met fin à une indépendance préservée depuis deux mille ans, un tiers de l'histoire de l'humanité. Elle réduit l'Écosse à un état de servitude. Cela est d'autant plus absurde que cette nation n'a pas été conquise par les

1. Il s'agit de An Account of a Conversation Concerning a Right Regulation of Governments for the Common Good of Mankind (Édimbourg, 1704).

2. James Hodges, The Rights and Interests of the Two British Monarchies... Treatise III (Londres, 1706) 66, 75, 81, 112; George Ridpath, Considerations upon the Union of the Two Kingdoms (Édimbourg, 1706) 48-49, 66. 3. Hodges 11, 16; Ridpath 50.

4. Robert Wyllie, A Letter Concerning the Union (Édimbourg, 1706) 6 .

5. Adopté par la Convention des États le 11 avril 1689, celui-ci prévoit notamment que le Parlement soit autorisé à se réunir régulièrement et que sa liberté d'expression ne soit pas entravée. armes. L'Union constitue une trahison envers tous ceux qui, par le passé, ont versé leur sang pour sauvegarder l'indépendance nationale. En la sacrifiant, les Écossais mettent en danger leurs libertés individuelles ${ }^{2}$. Les polémistes anti-unionistes semblent oublier que ces libertés ont souvent été moins bien respectées en Écosse qu'en Angleterre. Selon Hodges et Ridpath, l'Union est une sujétion: l'Écosse perd son Parlement et sa constitution; pendant ce temps, l'Angleterre garde les siens ${ }^{3}$. Le Parlement britannique n'est rien d'autre que le Parlement anglais avec quelques représentants en plus, constate Robert Wyllie, et la constitution britannique ne differre en rien de la constitution anglaise. L'union proposée est un marché de dupes ${ }^{4}$. D'ailleurs, les parlementaires écossais ne sont pas habilités à la conclure. Les pamphlétaires anti-unionistes sont unanimes sur ce point. D'après Hodges et Ridpath, l'Union est incompatible avec le Claim of Right ${ }^{5}$ et avec la CXXX ${ }^{e}$ loi du $\mathrm{VIII}^{\mathrm{e}}$ parlement de Jacques VI, qui qualifie de haute trahison toute tentative pour limiter les prérogatives des lords, des 
barons et des représentants des bourgs qui siègent au Parlement écossais ${ }^{6}$. Par ailleurs, en supprimant le Parlement d'Édimbourg, l'Union enfreint le droit des électeurs écossais à être représentés au sein d'une assemblée nationale. Elle ne peut avoir lieu que si tous les électeurs, sans exception, acceptent de se départir de leur droit de vote. Selon Hodges, le consentement de la totalité des épouses des électeurs est également indispensable. En fait, les auteurs anti-unionistes exhument les arguments avancés par Sir George Mackenzie et SirJohn Nisbet pour faire avorter un projet d'union antérieur. Du reste, Ridpath et Wyllie citent longuement ces deux éminents jurisconsultes. Patrick Abercromby invoque pour sa part un argument plus philosophique que juridique. Il utilise à ses propres fins le concept de souveraineté populaire exposé par Defoe dans The Original Power of the Collective Body of the People of England (1702): le Parlement, qui est subordonné au peuple, ne peut conclure l'Union contre son avis ; le peuple écossais a montré par ses pétitions qu'il était hostile à cette mesure; le Parlement n'est donc pas habilité à entériner l'Union ${ }^{7}$.

S'ils sont amenés à minimiser la souveraineté du Parlement écossais, les pamphlétaires unionistes tendent en revanche à créditer le futur Parlement britannique d'une souveraineté sans limite. D'après Hodges et Ridpath, rien n'oblige le futur Parlement de Grande-Bretagne à respecter les articles du traité d'Union. Il est même probable qu'ils les enfreindront et qưiils opprimeront l'Écosse. Noyés au milieu de 513 parlementaires anglais et gallois, les 45 représentants écossais ne pourront défendre les intérêts de leurs électeurs. En cas d'oppression - inévitable - de l'Écosse par le Parlement britannique, aucun recours n'est possible. Selon Ridpath, il est vain d'imaginer qu'une infraction au traité conduira automatiquement à l'annulation de l'Union, et que l'Écosse retrouvera sa liberté ${ }^{8}$. Abercromby s'inscrit également en faux contre une interpréta-

6. Hodges 72-74;

Ridpath V.

7. Patrick Abercromby,

The Advantages of the Act of Security, Compar'd with those of the Intended Union: Founded on the Revolution Principles Publish'd by Mr. Daniel De Foe (Édimbourg, 1706) 7-11.

8. Hodges 72,82 ; Ridpath 64, 69-70.

9. Abercromby 10, 13-16. tion lockienne du traité d'Union. Ce n'est pas parce que le Parlement porte atteinte au traité qu'il sera ipso facto frappé de dissolution. Les Parlements anglais et écossais ont tous deux abrogé nombre de lois aussi fondamentales que l'Act of Union sans mettre en péril leur existence.

Pour Abercromby, la souveraineté absolue du Parlement britannique est incompatible avec la sécurité de l'Église d'Écosse ${ }^{9}$. Livrée au bon vouloir du Parlement de Grande-Bretagne dominé par les anglicans, l'Église d'Écosse est condam- 
née à perdre son statut d'Eglise établie: l'Eglise est en danger!- un slogan paradoxalement emprunté aux anglicans ultras. Mais pour Webster, le sort de la Kirk n'est pas la seule source d'inquiétude. L'Union est incompatible avec les Covenants de 1638 et de 1643. Alors que les Écossais, par ces serments nationaux, s'engagent à réformer l'Église d'Angleterre et à supprimer son épiscopat, ils reconnaissent, par l'Union, la légitimité de l'Église d'Angleterre et de ses évêques. Ils commettent un parjure, et devront répondre de ce crime devant Dieux ${ }^{10}$. Hodges menace également de représailles divines ses concitoyens, coupables, à ses yeux, de s'unir à une nation pécheresse. S'il est moins pointilleux que Webster sur le chapitre des Covenants, il constate tout de même que ceux-ci ne peuvent s'accommoder de l'Union: l'existence de serments nationaux présuppose l'existence d'une nation; or l'Union sonne le glas de la nation écossaise ${ }^{11}$.

Celle-ci, d'après Hodges et Wright, a été sacrifiée au profit d'avantages commerciaux insignifiants. Si l'on en croit ces auteurs, la suppression des barrières douanières angloécossaises sera même préjudiciable à l'Écosse, qui importera davantage d'articles de luxe fabriqués à Londres et verra ses produits concurrencés par les produits anglais, plus compétitifs. Quant au commerce colonial, ce n'est qu'un leurre. Il sera impossible de réexporter les produits importés d'Amérique. Le marché européen est saturé. En tout état de cause, il se trouve déjà aux mains des Anglais ${ }^{12}$. Mais au fond, peu importe! Selon William Black, l'Écosse a les moyens de résoudre ses difficultés

10. James Webster, Laweful Prejudices against an Incorporating Union with England (Édimbourg, 1707) 4-6.

11. Hodges $47-52,57$.

12. Hodges $36-37,75$;

William Wright, The Comical History of the Mariage [sic] betwixt Fergusia and Heptarchus (Édimbourg, 1707), 18-19, 22.

13. William Black, Some Considerations in Relation to Trade (Édimbourg, 1706), 3-7; A Short View of our Present Trade and Taxes, Compared with what these Taxes may Amount to after the Union (Édimbourg, 1706), 2-8; Ridpath 72; John Hamilton, Lord Belhaven, The Lord Beilhaven's speech (Édimbourg, 1706), 4. économiques sans l'aide de l'Angleterre. Pour cela, elle doit améliorer la qualité de ses produits manufacturés, développer les différents secteurs existants de son commerce extérieur et consentir à limiter ses importations d'articles de luxe. Black a parfaitement intégré le dogme mercantiliste en cours au début du XVIII' siècle, mais il omet de signaler que les remèdes qu'il préconise sont appliqués depuis des décennies sans succès. Black, comme Ridpath, estime que la politique économique de l'Écosse ne saurait être dictée par le Parlement britannique. L'Écosse doit garder un certain contrôle sur son commerce. Black craint que l'augmentation de la pression fiscale inhérente à l'Union ne porte préjudice à l'économie écossaise. À l'instar de Belhaven, il dénonce les taxes qui pèseront sur le sel et la bière, et les répercussions prévisibles sur le prix de ces denrées ${ }^{13}$. Il se pose ainsi en défenseur des intérêts des 
consommateurs. Hodges et Wright soupçonnent que le revenu de ces taxes ne soit envoyé à Londres ${ }^{14}$. Comme Fletcher, ils craignent que la capitale n'absorbe toute la richesse de l'Écosse ${ }^{15}$. Londres, les anti-unionistes sont unanimes sur ce point, attirera aussi nombre d'Écossais. L'exode amorcé en 1603, dans le sillage de l'Union des couronnes, s'intensifiera si l'Écosse, après avoir perdu sa Cour, ne parvient pas à sauvegarder son Parlement. Soucieux de préserver cette institution, mais en même temps conscients des dysfonctionnements de l'Union des couronnes, les pamphlétaires anti-unionistes - à l'exception notoire d'Abercromby, qui souhaite ressusciter la «Vieille Alliance» avec la France ${ }^{16}$ - ne sont pas hostiles à une union fédérale ${ }^{17}$.

Leurs rivaux unionistes, en revanche, rejettent toute idée d'«union par fédération». Aucun projet concret d'union fédérale n'a été exposé à ce jour. Hodges n'a toujours pas publié le sien. En tout état de cause, l'Angleterre n'est pas disposée à conclure une telle union, qui est contraire à ses intérêts. Si l'Écosse veut obtenir le droit de commercer avec l'Angleterre et ses colonies, elle doit renoncer à sa souveraineté. Le sacrifice

14. Hodges $37-38$; Wright $22,25$.

15. Hodges 37-38; Wright 22, 25; Fletcher, Account of a Conversation, in Andrew Fletcher. Political Works 190, 212-213.

16. Abercromby $30-35$.

17. Voir, par exemple, Ridpath V; Hodges 119;

Anon., State of the Controversy betwixt United and Separate Parliaments (Édimbourg, 1706) 3, 27-28.

18. John Clerk, $A$ Letter to a Friend, Giving an Account how the Treaty of Union has been Received here (Édimbourg, 1706), 4, 42-44; William Seton, Scotland's Great Advantages by an Union with England (Édimbourg, 1706) 7-11; George Mackenzie, Lord Cromarty, Letters Concerning the Present Union from a Peer in Scotland to a Peer in England (Édimbourg, 1706) 6, 16, 26. 19. Le Parlement intérimaire qui donne une sanction législative à la Glorieuse Révolution en Écosse. n'est pas si grand qu'il y paraît. Seton, Cromarty et Glerk sont d'accord sur ce point : à présent, la souveraineté écossaise est purement factice; l'Écosse est sous l'influence de l'Angleterre; elle est gouvernée par des courtisans qui prennent leurs ordres à Londres; grâce à l'Union, l'Écosse mettra fin à cette situation de dépendance; la nation qu'elle formera avec l'Angleterre - la Grande-Bretagne - jouira d'une souveraineté absolue; elle sera puissante et pourra jouer un rôle important dans les affaires de l'Europe; en renonçant à sa souveraineté nominale, l'Écosse retrouvera, au sein de la Grande-Bretagne, une souveraineté et un pouvoir authentiques ${ }^{18}$. L'argument est habile. Toutefois, les unionistes omettent de signaler qu'en s'unissant à l'Angleterre, l'Écosse perd sa capacité de se gouverner sans en référer à une autre nation, qui est la caractéristique principale de la souveraineté. Les pamphlétaires unionistes affirment que le Parlement écossais est habilité à conclure l'Union. Selon Clerk, l'Union est conciliable avec le Claim of Right. La Convention des États ${ }^{19}$ n'a-t-elle pas recommandé une union des Parlements anglais et écossais sitôt après avoir voté la Déclaration des droits écossaise, en 1689? L’Union est également compatible avec la $\mathrm{CXXX}^{\mathrm{e}}$ loi du VIII ${ }^{\mathrm{e}}$ parlement de Jacques VI. Certes, il est interdit de supprimer l'un des trois 
Etats qui constituent le Parlement écossais, mais il est possible de diminuer le nombre de représentants des comtés et des bourgs, et de les ajouter ensuite à une autre assemblée ${ }^{20}$. Defoe utilise un argument voisin. Le Parlement n'est pas autorisé à déposséder les électeurs écossais de leur droit à être représentés au sein d'une assemblée. Cependant, il peut modifier les conditions d'exercice de ce droit: diminuer ou augmenter le nombre de représentants. Defoe réfute également l'argument de Patrick Abercromby. Le peuple a le droit de s'opposer à l'Union si celle-ci viole ses droits fondamentaux. Toutefois, l'Union ne menace ni les lois, ni les libertés, ni la religion, ni la propriété du peuple écossais. Celui-ci doit donc s'en remettre aux décisions de la législature suprême ${ }^{21}$. Seton affirme avec plus de vigueur encore la souveraineté du Parlement écossais. Seul juge des intérêts de la nation, le Parlement d'Édimbourg n'est assujetti à aucune autorité humaine. Il peut voter et abroger les lois comme bon lui semble. Seton rejette l'argument de Nisbet et de Mackenzie. Aucun texte juridique ne stipule que les lois fondamentales du royaume - à supposer que de telles lois existent - ne peuvent être abrogées sans le consentement de tous les électeurs. Le Parlement écossais est souverain. Bien entendu, il est tenu de respecter les lois naturelles - la liberté et la propriété-, mais l'Union ne met ni l'une ni l'autre en danger. Ayant défendu de manière aussi catégorique le concept de suprématie parlementaire, Seton est quelque peu embarrassé pour prouver que le futur Parlement britannique respectera les articles du traité d'Union et ne cherchera pas à opprimer l'Écosse. Il tente tout de même de rassurer ses compatriotes

20. Clerk, Letter, 13-14.

21. Daniel Defoe, $A$ Fourth Essay at Removing National Prejudices (Édimbourg, 1706) 10-12; A Fifh Essay at Removing National Prejudices (Édimbourg, 1706), 2-4.

22. William Seton, $A$ speech in Parliament Spoken by $\mathrm{Mr}$ Seton Junior of Pitmedden, November 181706 , in Daniel Defoe, The History of the Union of England and Scotland (Édimbourg, 1709) Part V, 76-78; Advantages 11.

23. Cromarty, Letters 8 , 23-24.

24. Hodges 49 .

25. Clerk 15, 37-38. par le truchement d'une métaphore organique: après l'Union, l'Écosse et l'Angleterre ne formeront plus deux corps, mais un seul $^{22}$. Cromarty ne cesse de s'étonner que les adversaires de l'Union puissent nier cette évidence ${ }^{23}$. L'hypothèse du suicidepratique, il est vrai, moins répandue en Écosse qu'en Angleterre, si l'on en croit Hodges ${ }^{24}$ - ne semble pas l'avoir effleuré. Clerk estime, lui aussi, que le Parlement britannique respectera les articles d'Union: ils sont équitables, et les deux nations ont intérêt à les observer. Il met toutefois ses lecteurs en garde: le Parlement britannique est une législature souveraine; rien, dans l'absolu, ne peut l'empêcher de violer les articles d'Union et d'opprimer l'Écosse ${ }^{25}$. Defoe, sur ce point, n'est pas d'accord avec son ami Clerk. Contrairement aux pamphlétaires unionistes écossais, il considère que la souveraineté du futur Parle- 
26. Celui-ci prévoit en particulier que le culte, la discipline et le gouvernement de l'Église d'Écosse ne pourront être modifiés par le Parlement britannique.

27. Daniel Defoe, An Essay at Removing National Prejudices against a Union with England. Part III (Édimbourg, 1706) 12-13, 26-27; Fourth Essay 8.

28. Seton, Advantages 4, 89; Cromarty 4; Defoe, Fourth Essay 6-7.

29. Daniel Defoe, Two Great Questions Considered. . Being a Sixth Essay at Removing National Prejudices against the Union (Édimbourg, 1707) 21-24.

30. John Arbuthnot, A Sermon Preach'd to the People, at the Mercat-Cross of Edinburgh, on the Subject of the Union (Edimburgh, 1706), 4-5, 18-20.

31. Considerations in Relation to Trade Considered (Édimbourg, 1706) 24;A Letter from Mr Reason 6.

32. Seton, Advantages 7-12; Clerk, Letter 17-22; Arbuthnot 16 ; Defoe, Fourth Essay 29-31; Fifth Essay 9-29. ment de Grande-Bretagne n'est pas sans limite. Le traité d'Union, pour autant qu'il donne naissance au Parlement britannique, lui est supérieur. Si le Parlement enfreint le traité, il ébranle du même coup ses fondations et se dissout.

Cet argument d'inspiration lockienne s'applique sans difficulté au cas de l'Église d'Écosse. L'Act of Security ${ }^{26}$, qui garantit la sécurité de l'Église, fait partie intégrante du traité d'Union. Par conséquent, le Parlement ne peut abroger l'Act of Security sans violer le traité et provoquer sa propre dissolution ${ }^{27}$. L'argumentation de Defoe repose sur une interprétation discutable du traité d'Union, élevé au rang de loi fondamentale. Mais elle a le mérite d'offrir aux presbytériens écossais une réponse cohérente et rassurante à leurs inquiétudes. Seton et Cromarty, tous deux épiscopaliens, sont silencieux sur le chapitre des garanties offertes à la Kirk. Cependant, ils soulignent, tout comme Defoe, que l'Union ne peut manquer de renforcer l'intérêt protestant contre la France catholique ${ }^{28}$. Defoe tente également de dissiper l'inquiétude des néo-covenantaires. L'Union et les Covenants ne se situent pas sur le même plan: la première relève du domaine civil; les seconds, du domaine religieux. L'Union ne peut donc être en contradiction avec les Covenants ${ }^{29}$.

Arbuthnot est visiblement agacé par les scrupules de Webster et de ses pairs: leur fanatisme est bien peu chrétien. En revanche, il semble considérer que l'esprit chrétien peut s'accommoder du désir de s'enrichir. L'Union permettra aux Écossais de l'assouvir. Elle leur apportera prospérité et abondance ${ }^{30}$. Les pamphlétaires unionistes, Defoe en particulier, sont intarissables sur les avantages économiques de l'Union. L'Union stimulera l'agriculture: les pratiques agricoles anglaises seront étendues à l'Écosse; les terres incultes seront mises en valeur. La pêche attirera les capitaux anglais. Elle pourra rivaliser avec la pêche hollandaise et, à terme, la dépasser. L'Union relancera l'industrie languissante ${ }^{31}$. Le commerce, sérieusement ébranlé par le protectionnisme des États européens, bénéficiera également de l'Union. L'Écosse pourra exporter librement en Angleterre sa toile, son bétail et son charbon. Elle aura la possibilité d'écouler ses produits manufacturés-en particulier ses grosses toiles - en Amérique, et d'en rapporter du tabac et du sucre, qu'elle réexportera en Europe. Cette activité commerciale retrouvée engendrera une hausse générale du niveau de vie et mettra fin à l'émigration qui mine l'Écosse depuis l'Union des couronnes ${ }^{32}$. 
Ces arguments sont-ils sincères? Expriment-ils le point de vue de leurs auteurs sur l'Union ou sont-ils le fruit de plumes vénales? La plupart des hommes qui participent au débat sur l'Union ne sont pas désintéressés, comme l'ont montré William Ferguson et Patrick Riley. Cromarty et Defoe, par exemple, sont rétribués pour leurs services - scripturaires et autres - en faveur de l'Union. Cromarty reçoit $£ 300$ d'arrérages, versées par le Trésor écossais, auxquelles s'ajoutent sans doute $£$ $1500^{33}$, prélevées sur l'«équivalent »- le prêt de $£ 39808510 \mathrm{~s}$. octroyé par l'Angleterre à l'Écosse en contrepartie de sa souscription à la dette anglaise. Entre octobre et décembre 1706, Defoe perçoit la somme de $£ 103$ 8s. pour mener à bien la mission qui lui a été confiée par Harley. Il espère également être récompensé par un poste dans les douanes écossaises ${ }^{34}$. Contrairement à ce que suggère P.H. Scott ${ }^{35}$, les polémistes unionistes ne sont pas les seuls à être stipendiés. Hodges et Ridpath sont des pamphlétaires professionnels, qui travaillent contre rémunération. Ils sont, selon toute probabilité, à la solde du duc de Hamilton. Par ailleurs, même s'ils ne sont pas directement rétribués pour leurs écrits, plusieurs pamphlétaires prennent la plume par souci de promouvoir ou, du moins, de ne pas entraver leur carrière. Seton, qui, en 1704, a négocié son soutien au parti de la Cour contre une pension de $£ 100$ par an, ne peut refuser de défendre une cause que la Cour souhaite voir triompher. Clerk, qui est redevable de sa brillante carrière- en particulier de sa nomination dans plusieurs commissions royales - au duc de Queensberry, chargé de faire ratifier le traité d'Union par le Parlement écossais, se doit de prêter son concours à la cause unioniste. Il serait mal venu d'offenser un noble protecteur en manifestant une quelconque tiédeur. Quant à Arbuthnot, promu médecin extraordinaire de la reine Anne en 1705, il sait qu'en composant un pamphlet favorable à l'Union, il ne peut manquer d'obtenir la reconnaissance de son auguste patiente, désireuse d'unir plus étroitement ses deux

33. Historical Manuscripts Commission, Mar and Kellie Manuscripts (Mar à Nairne, 17 décembre 1706).

34. The Letters of Daniel Defoe, ed. George Harris Healey (Oxford: Clarendon Press, 1955) 223-224. 35. Voir, par exemple, Daniel Defoe in Edinburgh and Other Papers (Édimbourg: Tuckwell Press, 1995). royaumes.

Toutefois, il y a tout lieu de penser que certains pamphlétaires - anti-unionistes essentiellement - écrivent sans espoir de gratification. Robert Wyllie et William Wright, tous deux ministres presbytériens, prennent la plume pour dénoncer une union qui, à leurs yeux, menace la sécurité de l'Église d'Écosse et bafoue les Covenants. Patrick Abercromby, ancien médecin de Jacques II, compose un pamphlet afin de discréditer une 
36. Le comte de Mar est l'un des deux secrétaires d'État du gouvernement écossais.

37. H.M.C., Mar and Kellie (17 novembre 1705) 238; (1er janvier 1706) 242-243. 38. H.M.C., Mar and Kellie

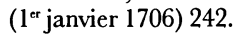

39. H.M.C., Mar and Kellie (Mar à Cromarty,

28 novembre 1705) 239;

(Mar à Godolphin, 16 septembre 1706) 278; (Seafield à Mar, 20 août 1706) 273 ; (Nairne à Mar, 5 octobre 1706) 285.

40. Letters of

Defoe (5 novembre 1706)

143; (13 novembre 1706)

146; (16 janvier 1707) 193.

41. Letters of Defoe

(28 décembre 1706) 187.

42. Letters of Defoe (Defoe à

Harley, 21 décembre 1706) 179. mesure préjudiciable à la cause jacobite. William Black, manufacturier de son état, veut mettre la communauté économique écossaise en garde contre les prévisions excessivement optimistes de Defoe et de ses pairs. Tous ces gens, qui ne sont pas des polémistes professionnels, agissent apparemment en francs-tireurs, pour défendre leurs convictions.

Par ailleurs, il semblerait qu'en définitive, les pamphlétaires stipendiés - ou inféodés - ne trahissent pas leur point de vue personnel sur l'Union. Cromartie, Defoe, Seton, Clerk et Arbuthnot sont, à des degrés divers, persuadés du bien-fondé de cette mesure. Cromarty, dans ses lettres au comte de $\mathrm{Mar}^{36}$, appelle l'Union de tous ses vœux. Celle-ci garantira la sécurité de la Grande-Bretagne et libérera l'Écosse des maux qui l'affligent. Cromarty rejette toute union fédérale. Seule l'union des Parlements désirée par Londres trouve grâce à ses yeux ${ }^{37}$. Il émet le souhait que les différentes marques de distinction entre Anglais et Écossais soient effacées: «May wee be Brittains and down goe the old ignominous names of Scotland, of England... Brittains is our true, our honorable denomination ${ }^{38}$. Le zèle unioniste de Cromarty est reconnu par Mar, Seafield et Sir David Nairne ${ }^{39}$. Il n'est pas remis en question par Fletcher, qui, dans An Account of a Conversation (1704), prête à Cromarty les arguments unionistes qu'il s'efforce de pourfendre.

Defoe, dans sa correspondance, exprime à plusieurs reprises son attachement à l'Union ${ }^{40}$. Ses déclarations doivent être considérées avec circonspection: Defoe ne s'adresse-t-il pas précisément à l'homme qui l'emploie pour promouvoir la politique unioniste du gouvernement anglais? En revanche, l'on ne saurait mettre sa parole en doute lorsqu'il assure à son ami John Fransham, drapier à Norwich, qu'il souhaite voir l'Union aboutir: "tis pity the two Nations should be divided any longer. This people are a Sober, Religious and Gallant Nation, the country good, the Soil in most places capable of vast improvements and nothing Wanting but English Stocks, English Art and English Trade to make us all one great people ${ }^{41}$. Seton, d'après Defoe, est, lui aussi, un unioniste sincère ${ }^{42}$. La constance de ses prises de position en faveur de l'Union depuis 1700 tend à confirmer ce jugement. Defoe, à n'en pas douter, aurait porté le même jugement sur son ami John Clerk. Dans son Testamentary Memorial, rédigé le 20 octobre 1744 à l'intention de ses proches, Clerk exprime à l'égard de l'Union une 
approbation sans réserve ${ }^{43}$. Son attitude en 1706-1707 est plus nuancée. Toutefois, les lettres qu'il envoie à son père pendant cette période montrent qu'il n'est pas hostile à l'Union ${ }^{44}$. Arbuthnot, à l'évidence, ne l'est pas davantage. Son ami, Lord Peterborough, s'adresse à lui comme à un unioniste sincère ${ }^{45}$. Cela, à juste titre, puisque, dans une lettre à Mar datée du $1^{\text {er }}$ février 1707, il souhaite que l'Union, après avoir été entérinée par le Parlement écossais, soit ratifiée par Westminster ${ }^{46}$.

Si Cromarty, Defoe et leurs alliés sont convaincus du bienfondé de l'Union, Ridpath est persuadé de sa nocivité. Lorsque le Nouveau parti avec lequel il entretient des relations privilégiées, décide de s'allier au parti de la Cour et de soutenir l'Union, il s'en désolidarise, et continue de défendre la cause anti-unioniste avec un zèle qui indispose fortement Mar et Nairne ${ }^{47}$.

43. A Testamentary Memorial Concerning the Union of the Two Kingdoms, of Scotland $\mathcal{E}^{\circ}$ England in 1707 with a Short Account of the Share I had in the Settlement of the Present Government of Great Britain, in Clerk, History 182-208 (Appendice C).

44. Clerk, History 3-4 (Introduction de Douglas Duncan).

45. Lettre de Lord Peterborough citée dans George A. Aitken, The Life and Works of John Arbuthnot, M.D., Fellow of the Royal College of Physicians (Oxford: Clarendon Press, 1892), 29-30.

46. H.M.C., Mar and Kellie (1 $1^{\text {er }}$ février 1707) 370.

47. H.M.C., Mar and Kellie (Nairne à Mar, 7 novembre 1706) 315.

48. Letters of Defoe (Defoe à Harley, 2 mars 1711) 319. 49. Letters of George Lockhar of Carnwath 1698-1732, ed. Daniel Szechi (Édimbourg: Pillans \& Wilson, 1989) 33 (Lockhart à

Maule, 26 mai 1706).

50. P.W.J. Riley, The

Union of England and Scotland: A Study in Anglo-Scottish Politics of the 18th Century (Manchester: Manchester University Press, 1978).

51. Letters of Defoe (Defoe à Harley, 18 mars 1707) 209210.
Les pamphlétaires sont-ils prêts à utiliser n'importe quel argument pour promouvoir leur point de vue sur l'Union? À l'évidence, leurs arguments ne sont pas toujours sincères. Abercromby déplore que l'Union mette l'Église d'Écosse en danger, alors qu'il est épiscopalien et que le sort de la Kirk lui est indifférent. Defoe fait grand cas des garanties juridiques offertes à l'Église d'Écosse par le traité d'Union. Toutefois, lorsqu'il converse avec les ministres presbytériens, il leur donne à entendre que la sécurité de la Kirk ne repose pas tant sur le traité que sur la parole solennelle de la reine Anne, qui a promis de la protéger ${ }^{48}$. Il affirme dans ses Essays que le Parlement d'Édimbourg n'est pas habilité à conclure l'Union contre l'avis du peuple écossais, alors que dans Legion's Memorial (1701) et dans The Original Power of the Collective Body of the People of England (1702), il défend le concept de souveraineté populaire. Seton ne laisse pas d'énumérer les avantages économiques de l'Union. Mais en privé, il confie à Lockhart qu'il n'approuve pas les clauses fiscales du traité ${ }^{49}$.

Toutefois, les pamphlétaires - y compris les unionistes - qui participent au débat sur l'Union ne sont pas aussi cyniques que le pense Patrick Riley ${ }^{50}$. Jacobite, et donc francophile, Abercromby est sincère quand il affirme que l’Écosse a intérêt à ressusciter la "Vieille Alliance» avec la France. Webster croit réellement que l'Union est incompatible avec les Covenants: lorsque Defoe tente de prouver le contraire dans son sixième Essay, il le traite de blasphémateur, l'accuse d'immoralité et lui interdit l'accès de son église ${ }^{51}$. Defoe, pour sa part, est 
convaincu que les clauses économiques du traité d'Union sont favorables à l'Écosse. Fletcher, explique-t-il dans l'une de ses lettres à Harley, s'est discrédité lorsqu'il a déclaré à la Chambre que le droit de commercer librement avec l'Angleterre n'était pas un avantage pour l'Écosse. Les parlementaires qui ont voté contre l'article IV - accordant aux Écossais les mêmes avantages commerciaux qu'aux Anglais - n'ont fait que desservir la cause unioniste ${ }^{52}$. Par ailleurs, l'on ne peut mettre en doute la sincérité des propos de John Clerk sur la question de la souveraineté écossaise. Avant l'Union, explique-t-il dans son Testamentary Memorial (1744), l'Écosse était une nation vénérable, mais pauvre. Son Parlement détenait seulement un pouvoir symbolique. Il se réunissait toujours sous la férule du gouvernement anglais et ne faisait qu'entériner les instructions de Londres. Mais, grâce à l'Union, l'Écosse a acquis une souveraineté, une autorité et une dignité égales à celles dont jouissait l'Angleterre avant $1707^{53}$.

Les pamphlétaires sont au moins en partie convaincus de la légitimité des arguments qu'ils avancent pour défendre leur point de vue sur l'Union. Réussissent-ils à convaincre leurs compatriotes? On sait que leurs écrits sont lus par les parlementaires, qui y puisent les arguments qu'ils utilisent dans leurs discours. On sait également que certains de ces écrits bénéficient d'une diffusion appréciable. D'après Defoe, The Rights and Interests of the Two British Monarchies... Treatise III l'ouvrage anti-unioniste de Hodges - circule «dans tout le royaume ${ }^{54}$. Dans une lettre du 9 décembre 1706, l'émissaire de Harley annonce son intention de diffuser l'un de ses pamphlets - A Letter to the Glasgow-Men - non seulement à Glasgow, mais dans tout l'ouest de l'Écosse : à Lanark, à Hamilton, à Stirling et à Dumfries. Il entend faire tirer cet opuscule à 2500 exemplaires ${ }^{55}$.

Cependant, l'influence réelle de ces écrits reste difficile à déterminer. Les pamphlétaires pèsent-ils de manière significative sur le vote des parlementaires? Contribuent-ils à enflammer - ou, dans certains cas, à apaiser - le petit peuple des villes

52. Letters of Defoe (Defoe à Harley, [22] novembre 1706) 153-154. 53. Clerk, Testamentary Memorial 186-187, 189, 206 54. Letters of Defoe (Defoe à John Fransham, 28 décembre 1706) 185. 55. Letters of Defoe, 170. et des campagnes écossaises? La réponse à la première question est négative. Sur les votes importants, les parlementaires suivent presque toujours la consigne de leur parti. Il ne faut pas tant y voir l'incapacité des polémistes à convaincre que la qualité de la discipline exercée par les partis en général, et par le parti de la Cour en particulier. 
56. Idem, 148.

57. Idem, 153.

58. Idem, 170 .

59. Idem, 153.

60. H.M.C., Mar and Kellie

(Mar à Nairne, 3 novembre 1706) 309.

61. H.M.C., Mar and Kellie (Mar à Nairne, 26 octobre 1706) 298; (Mar à Godolphin, 26 octobre 1706) 302.
L'influence des pamphlétaires sur le petit peuple est plus sensible. Le 14 novembre 1706, Defoe rapporte à Harley que sa réponse au discours de Belhaven - un petit poème satirique intitulé The Vision - a diverti certains cercles édimbourgeois et s'est sans doute avérée plus efficace qu'une œuvre plus sérieuse ${ }^{56}$. Le 22 novembre, il lui confie que son quatrième Essay a produit «un certain effet ${ }^{57}$. Le 12 décembre, il se félicite de l'accueil réservé à $A$ Letter to the Glasgow-Men: ce pamphlet a contribué à ramener le calme à Glasgow ${ }^{58}$. Ces propos ne peuvent être pris pour argent comptant. Defoe, à l'évidence, se vante pour impressionner son employeur. En revanche, il n'y a aucune raison de mettre sa parole en doute lorsqu'il évoque, dans sa lettre à Harley du 22 novembre, l'influence exercée par The Rights and Interests of the Two British Monarchies... Treatise III: Hodges, par son pamphlet, a davantage nui à l'Union que 1000 hommes réunis ${ }^{59}$. Ce jugement est d'ailleurs corroboré par Mar: «Hodge [s] has sent down a book here... it is right calculated to catch the ministers and comonalty; so really it has done harm " ${ }^{60}$. Toutefois, l'influence des pamphlétaires est inférieure à celle qu'exercent, depuis leurs chaires, les ministres presbytériens sur leurs ouailles ${ }^{61}$. Cela ne remet en cause ni la diligence, ni la compétence des polémistes. Tous les Écossais - ou presque - vont écouter leur pasteur à l'église paroissiale. Mais seulement les plus aisés et instruits d'entre eux peuvent lire et comprendre les pamphlets politiques publiés à Édimbourg ou à Londres. 